\title{
Preserved Drawing in a Sculptor with Dementia
}

\author{
Luis Fornazzari, Thom Ringer, Lee Ringer, Corinne E. Fischer
}

Can J Neurol Sci. 2013; 40: 736-737

Learning and recalling new pieces of music ${ }^{1}$ and preserved painting ${ }^{2}$ are reported in Alzheimer's disease (AD) and related dementias. In some cases of Frontotemporal Dementia (FTD), artistic creativity emerges as the disease develops ${ }^{3}$. It has been suggested that art and creativity may be subserved by distinct neural networks. In this clinical report, we explore these distinctions in a sculptor who continues drawing spontaneous sketches of faces and figurines, including from memory, in spite of advanced Vascular Dementia (VD), thus suggesting the persistence of creativity notwithstanding marked visuospatial and visual memory deficits as recorded in standard tests.

\section{Case Report}

Mary Hecht is a 76-year-old bilingual woman who resides in a long term care facility. She is a prolific and renowned sculptor who is highly educated, with a BA in sociology (1952) and a master's degree in sculpture (1957). We are revealing her identity in this manuscript as it was her expressed wish to have her identity known. She was assessed by our Geriatric Mental Health Outreach Team (GMHOT) for behavioural and psychological symptoms of dementia (BPSD) including depression, apathy, sleep difficulties, and verbal aggression towards staff.

She fulfilled the NINDS-AIREN and DSM IV- R criteria for probable Vascular Dementia (VD) and was wheelchair bound due to bilateral cerebellar and right side pontine strokes, which occurred two years before our assessment. Relevant medical illnesses included hypothyroidism, hypertension, depression, and chronic obstructive pulmonary disease. On neurological assessment, she was not able to stand up by herself and to keep the standing position when assisted.

She had midline cerebellar ataxia and mild right-sided weakness with decreased muscle tone and slightly increased tendon jerk reflexes on the right side. All her cranial nerves, sensory responses to touch, pinprick, joint position, vibration sense, and right and left graphaesthesias were normal. Her brain magnetic resonance imaging (MRI) confirmed moderate to severe generalized brain and cerebellar atrophy, extensive periventricular microangiopathic hyperintensities and old strokes in the right pons as well as both cerebellar hemispheres.

At initial assessment, her scores on the Montreal Cognitive Assessment and Minimental Status Examination were 12/30 and $14 / 30$ respectively. Her specific subscores in the MOCA were visuospatial 0/2, the Clock Drawing test (CDT) 0/3, naming 3/3, attention $6 / 6$, language $2 / 2$, orientation $1 / 6$, abstraction $0 / 2$, and memory $0 / 5$. Her reproduction of the Rey-Osterreich complex figure was distorted, incomplete and barely recognizable, with a total score of $12 / 36$, indicating impaired perceptual organization and visual memory. Her time to perform Trail Making Test A

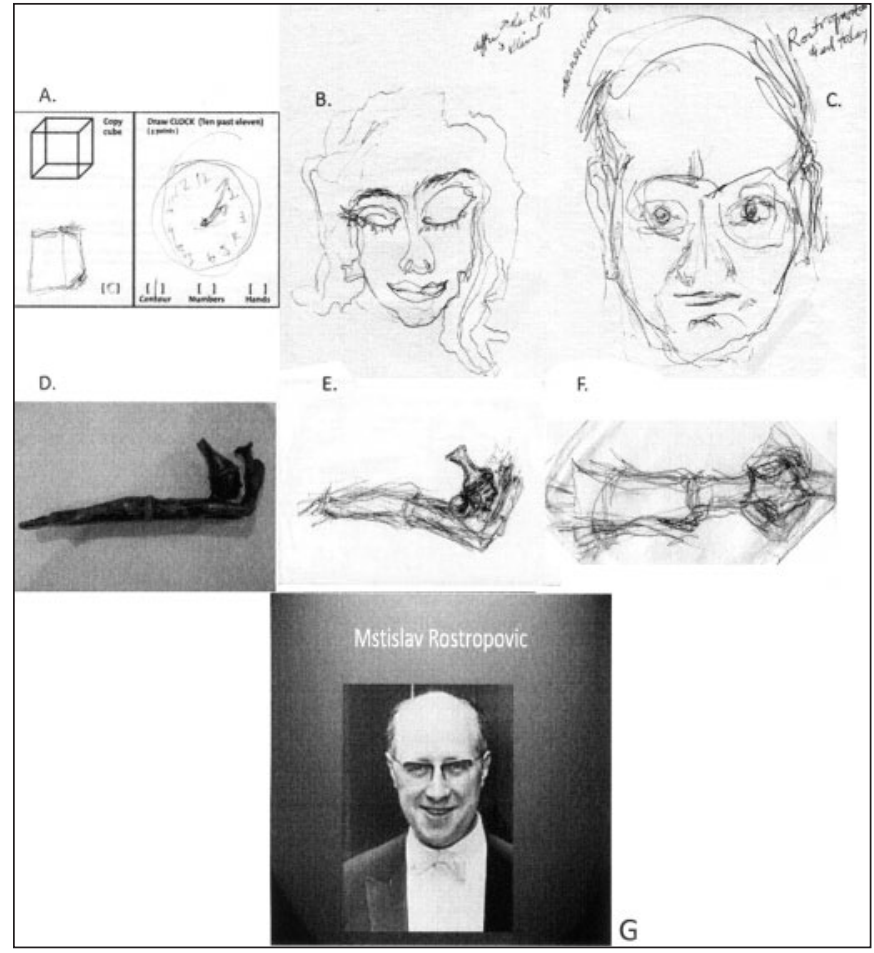

Figure 1: A. Impaired copy of cube and Clock Drawing Test; B. Spontaneous sketch of a student; $C$. Spontaneous sketch of D Rostropovich; D. Lyng Buddha Sculpture; E. Copy of lying Buddha; F. Recall of Buddha 10 minutes later; G. Photography of D Rostropovich

(TMT-A) was three minutes. She abandoned TMT-B after two minutes and 50 seconds. Her spontaneous language was well preserved and rich. She took great pleasure in showing and discussing her art using a vast, elaborate vocabulary, sophisticated metaphors and figures of speech.

While her spontaneous CDT and copying of the cube and the two overlapping pentagons were done very poorly (Figure 1A), she was able to produce a freehand sketch of a lying Buddha figurine, and reproduce the sketch from memory ten minutes later (Figures 1D, E, F). She also produced an attractive sketch

From the St. Michael's Hospital, Department of Psychiatry, Memory Disorders Clinic (LF, TR, LR, CEF), Department of Medicine, Division of Behavioural Neurology (LF, Department of Psychiatry (LF, CEF), University of Toronto, Toronto, Ontario, Canada. Received December 3, 2012. Final Revisions Submitted April 11, 2013. Correspondence to: Luis Fornazzari, 27 Cavendish St Toronto Ontario, M4E 1N9, Canada.Email: forna@rogers.com. 
of our research student (Figure 1B). Perhaps most impressively, she spontaneously drew an accurate sketch of acclaimed cellist Mitislav Rostropovic as she had known him some 20 years earlier and whose death she had just learned of that day on the radio (Figure $1 \mathrm{C}$ ).

We have continued to see the patient every six months for routine follow up. Her irritability, verbal aggression, and sleep difficulties have improved, but her cognitive deficits have continued to progress. She cannot recognize any member of our team and is no longer testable on conventional neuropsychological tests. She is not drawing, even when invited to do so.

\section{Discussion}

Our patient's excellent drawing and copying capabilities, despite marked visuospatial and visual memory deficits on standard tests, suggest the persistence of her art and creativity in the context of an ongoing VD. This may be explained by the relative preservation of the right and left posterior parietal lobes, which in her case show diffuse atrophy. While right hemisphere functions are associated with copying images, retrieving imagery, drawing real images, and cross-association sensations to produce art, left hemisphere functions play a role in art, including development of symbolism, abstraction, and transformation of linguistic concepts into other forms of arts expression ${ }^{4}$. Although copying is a highly visual task requiring frequent shifts of spatial attention, drawing from memory requires more constant attention and access to memory retrieval systems in normal volunteers ${ }^{5}$. Our patient's performance on standard cognitive and specific neuropsychological tests demonstrates her impairment in the above domains. Art skills may be protected when other brain functions are declining, as reported in AD, FTD, and as in our patient with VD.

\section{REFERENCES}

1. Fornazzari L, Castle T, Nadkarni S, et al. Preservation of episodic musical memory in a pianist with Alzheimer's disease. Neurology. 2006;66:610-11.

2. Fornazzari L. Preserved painting creativity in an artist with Alzheimer's disease. Eur J Neurol. 2005; 12:419-24.

3. Miller BL, Hou CE. Portraits of artists: emergence of visual creativity in dementia. Arch Neurol. 2004;61:842-4.

4. Cummings JL, Miller BL, Christensen DD, et al. Creativity and dementia: emerging diagnostic and treatment methods for Alzheimer's disease. CNS Spectr. 2008;13(2 suppl 2):1-20.

5. Ferber S, Mraz R, Baker N, et al. Shared and differential neural substrates of copying versus drawing: a functional magnetic resonance imaging study. Neuro Report. 2007;18:11:1089-93. 